\title{
Can we predict lymph node metastasis by using preoperative markers in gastric cancer patients?
}

\author{
Mateusz Pryt, Marta Kalwas, Dariusz Nejc, Arkadiusz Jeziorski
}

Department of Surgical Oncology, Medical University of Łódź, Poland

Introduction. Lymph node involvement is a negative prognostic factor for patients undergoing curative gastrectomy and indication for preoperative chemotherapy, thus a sufficient assessment strategy is required. We aimed to establish the value of preoperatively collected data in prediction of lymph node metastasis.

Material and methods. We conducted a retrospective analysis of 150 gastric cancer patients hospitalized in the Department of Surgical Oncology, Medical University of Łódź in 2011-2017. We gathered information comprising clinicopathological features, inflammatory markers: neutrophil to lymphocyte ratio (NLR), platelet to lymphocyte ratio (PLR), systemic inflammatory response index (SIRI) and Tumor Index (TI).

Results. Independent factors associated with lymph node involvement were: NLR (OR = 1.35; $p=0.01)$, Tumor Index $(O R=1.07 ; p<0.001)$. ROC curve plots assessed the diagnostic value of TI and NLR.

Conclusions. Tumor Index and NLR are factors indicating lymph node metastasis in gastric cancer patients. NLR is a potentially useful tool for neoadjuvant chemotherapy qualification.

NOWOTWORY J Oncol 2019; 69, 1: 7-11

Key words: gastric cancer, neutrophil/lymphocyte ratio, platelet/lymphocyte ratio, lymph node metastasis

\section{Introduction}

Despite consistent decrease in incidence and mortality, gastric cancer remains the third leading cause of cancer death worldwide. Five-year survival ranges between $20 \%$ and $70 \%$ in different regions of the world [1,2]. Poor prognosis and lack of targeted therapies make surgery still the main and the most important treatment option [3, 4]. Due to high recurrence rate, even after curative resection, adjuvant therapy is a part of routine treatment [2].

Recently, several trials revealed that neoadjuvant chemotherapy improves patient survival [5-8]. Combination of pre- and postoperative chemotherapy is recommended in advanced stages, especially those with lymph node metastases $[3,4,9]$. Wide range of tools and techniques for lymph node assessment, such as endoscopic ultrasonography, computed tomography or MRI is available, but their clinical value is questionable because of their inconsistent results [4, 10-12].
Several biomarkers connected with lymph node metastases have been considered, however, up to date none of them have proven their usefulness in routine clinical practice. The role of systemic inflammatory response and tumor microenvironment in cancer progression and promotion of metastasis has been widely explored [13, 14]. Leukocyte infiltration and inflammation is the key part of physiological response to tumor growth in gastric cancer $[15,16]$. The moment when tumor factors overcome systemic response and affect cytokine and chemokine production, together with leukocyte differentiation, is probably when the growing neoplasm gains metastatic ability [17-20]. This complex interaction is reflected in peripheral blood count and acute-phase proteins level. Several systemic inflammatory markers, including neutrophil-to-lymphocyte ratio (NLR), platelet-to-lymphocyte ratio (PLR), monocyte-to-lymphocyte ratio (MLR) and Systematic Inflammatory Response Index (SIRI), have been established 
as independent prognostic factors in many different cancers (21-25). Tumor Index (TI) is a simple indicator, which combines depth and size of primary tumors [26]. While prognostic value in gastrointestinal neoplasms is widely explored, only few studies examined the role of preoperative markers in assessment of lymph node status $[24,25]$. In this study we aimed to establish the value of preoperatively collected data: previously mentioned markers and clinicopathological data as preoperative predictors of lymph node metastasis in patients undergoing curative surgery for gastric cancer.

\section{Material and methods}

\section{Patients, collected data and inclusion criteria}

We conducted a retrospective analysis of 350 consecutive gastric cancer patients hospitalized in the Department of Surgical Oncology, Medical University of Łódź, between January 2011 and December 2017; 150 of them underwent elective curative gastrectomy and fulfilled the study criteria. Out of the excluded patients, 139 accounted for non-curative resections, 35 underwent neoadjuvant treatment, 5 had less than 15 harvested lymph nodes and in 6 patients postoperative histological evaluation did not confirm adenocarcinoma diagnosis. Additionally, 13 patients presented with chronic and 2 with acute inflammatory diseases, which could influence laboratory blood test results. We gathered information comprising age, sex, tumor histopathological features, size and location, TNM stage, blood test results and course this of medical treatment. Histological type was assessed according to Lauren classification (intestinal, diffuse and mixed type) and according to WHO classification of gastric tumors [8]. Patient characteristics are shown in Table I.

\section{Definition of calculated markers}

Laboratory measurements comprised full blood count (erythrocytes, platelets, neutrophils, lymphocytes, monocytes) and were performed 1-2 days before the surgery. Neutrophil to lymphocyte ratio (NLR), platelet to lymphocyte ratio (PLR), monocyte to lymphocyte ratio (MLR), systemic inflammatory response index (SIRI = neutrophil count $\times$ monocyte count/ lymphocyte count) and Tumor Index ( $\mathrm{TI}=\mathrm{T}$ from TNM stage category $\times$ tumor size in $\mathrm{cm}$ ) were obtained.

\section{Statistical analysis}

We performed statistical analysis using SPSS 23. Univariate analysis was done to reveal statistically significant factors indicating the presence of lymph node metastasis. Distribution normality of continuous variables was evaluated by Shapiro-Wilk test. The difference between lymph node positive and lymph node negative groups in categorical variables was assessed using chi-squared test, in continuous normally distributed variables using t-test and in continuous non-normally distributed variables using Mann-Whitney U test. P value $<0.05$
Table I. Patient characteristics

\begin{tabular}{|c|c|}
\hline Parameter & Number of patients (\%) \\
\hline \multicolumn{2}{|l|}{ Age at the time of surgery } \\
\hline Median age $( \pm$ SD) & $(65 \pm 10.1)$ \\
\hline \multicolumn{2}{|l|}{ Patient sex } \\
\hline Female & $56(37.4 \%)$ \\
\hline Male & $94(62.6 \%)$ \\
\hline \multicolumn{2}{|l|}{ Tumor stage } \\
\hline $\mathrm{T} 1$ & $25(16.7 \%)$ \\
\hline $\mathrm{T} 2$ & $19(12.7 \%)$ \\
\hline $\mathrm{T} 3$ & $75(50 \%)$ \\
\hline $\mathrm{T} 4$ & $31(20.6 \%)$ \\
\hline \multicolumn{2}{|l|}{ Lymph node involvement } \\
\hline NO & $47(38.2 \%)$ \\
\hline N1 & $16(13 \%)$ \\
\hline N2 & $21(17.1 \%)$ \\
\hline N3 & $39(31.7 \%)$ \\
\hline \multicolumn{2}{|l|}{ Lauren classification } \\
\hline Intestinal type & $69(46 \%)$ \\
\hline Diffuse type & $50(33.3 \%)$ \\
\hline Mixed type & $31(20.7 \%)$ \\
\hline \multicolumn{2}{|l|}{ WHO Classification } \\
\hline Tubular type & $63(42 \%)$ \\
\hline Poorly cohesive carcinoma & $55(36.7 \%)$ \\
\hline Other & $32(21.3 \%)$ \\
\hline \multicolumn{2}{|l|}{ Localization } \\
\hline Body & $92(54.7 \%)$ \\
\hline Pylorus & $47(31 \%)$ \\
\hline Cardia & $7(4.7 \%)$ \\
\hline Major part of the stomach & $14(9.3 \%)$ \\
\hline \multicolumn{2}{|l|}{ Tumor size } \\
\hline $\begin{array}{l}\text { Median size } \\
\text { mean }( \pm S D)\end{array}$ & $\begin{array}{c}4.9 \\
(5.05 \pm 2.6)\end{array}$ \\
\hline \multicolumn{2}{|l|}{ Lymph node yield } \\
\hline $\begin{array}{l}\text { Median yield } \\
\text { mean }( \pm S D)\end{array}$ & $\begin{array}{c}26 \\
(27.8 \pm 10.3)\end{array}$ \\
\hline
\end{tabular}

from univariate analysis was a criterion to include a variable in multivariate logistic regression to assess which of them were independent factors. We checked our model to meet key logistic regression assumptions: the observations were independent, there was no correlation between variables, the independent variables were linearly related to the log odds.

Receiver operating characteristic curves (ROC) were obtained for the statistically significant continuous predictors. Sensitivity, specificity, positive and negative predictive values were calculated. Optimal cutoff values for ROC curves were calculated using Youden Index (sensitivity + specificity-1) [27]. $P$ value $<0.05$ was considered statistically significant. 


\section{Results}

\section{Univariate and multivariate analysis of clinicopathological characteristic and obtained markers}

Out of the considered variables NLR $(p=0.004)$, SIRI ( $p=0.02)$, T feature according to TNM staging system $(p=0.001)$, Tumor Index $(p<0.001)$ and Lauren mixed type $(p=0.008)$ showed association with the presence of lymph node metastasis (Tab. II).

There was no impact of age $(p=0.907)$, sex $(p=0.132)$, tumor size $(p=0.153)$, lymph node yield $(p=0.107)$, grading $(p=0.23)$, any blood cell count, PLR $(p=0.203)$ or MLR $(p=0.388)$ on lymph node status. Due to correlation and lack of independency between T stage and Tumor Index and between NLR and SIRI, we decided not to include T stage and SIRI in the multivariate analysis. NLR and TI as predictors explained more variation in lymph node status. The remaining statistically significant factors were included.

Multivariate logistic regression revealed that independent factors associated with the presence of lymph node metastasis were: NLR with $\mathrm{OR}=1.35 ; 95 \% \mathrm{Cl}[1.08-1.7] ; \mathrm{p}=0.009$, Tumor Index (TI) with $\mathrm{OR}=1.41 ; 95 \% \mathrm{Cl}[1.2-1.7] ; \mathrm{p}<0.004$ and mixed-type by Lauren with $\mathrm{OR}=3.85 ; 95 \% \mathrm{Cl}[1.12-13.2]$; $p=0.033$.

\section{ROC curves and clinical value of markers}

ROC curve analysis allowed to assess the diagnostic value of Tumor Index ( $A \cup C=0.66 ; p<0.001$ ), cutoff point -6 and NLR ( $A \cup C=0.63 ; p=0.002$ ), cutoff point -4.1 . For TI, in our group 113 (75.3\%) patients were above the mentioned cutoff point. 80 of them had lymph node metastasis reported in postoperative histopathological exam. Out of 37 patients below the cutoff point, 12 (37\%) had lymph node involvement. This translates into $87 \%$ sensitivity, $43 \%$ specificity, $71 \%$ positive predictive value (PPV) and $67 \%$ negative predictive value (NPV). For NLR cutoff value of 4.1 , associated with 35\% sensitivity, 90\% specificity, $84 \%$ positive predictive value (PPV) and $46 \%$ negative predictive value (NPV).

\section{Discussion}

Gastric cancer is a major public health concern worldwide. Because of unspecific symptoms and screening programs performed only in several Asian countries, stomach malignancies are usually diagnosed at advanced stages. In Europe surgery with D2 lymphadenectomy is a treatment-of-choice, however, surgery alone is associated with poor prognosis and high local recurrence rate $[3,4]$. Therefore, various adjuvant treatment options have been developed, with postoperative chemoradiotherapy or chemotherapy alone being a treatment-of-choice for most patients after curative resection. Recently neoadjuvant chemotherapy is widely advocated, showing a survival benefit compared with surgery alone, with similar morbidity rates (2.5-9.28). Perioperative treatment, consisting of pre- and postoperative chemotherapy is becoming treatment-of-choice for most patients diagnosed with gastric cancer at an advanced stage, resulting in overall survival benefit $[3,9]$. However, neoadjuvant treatment seems to be the

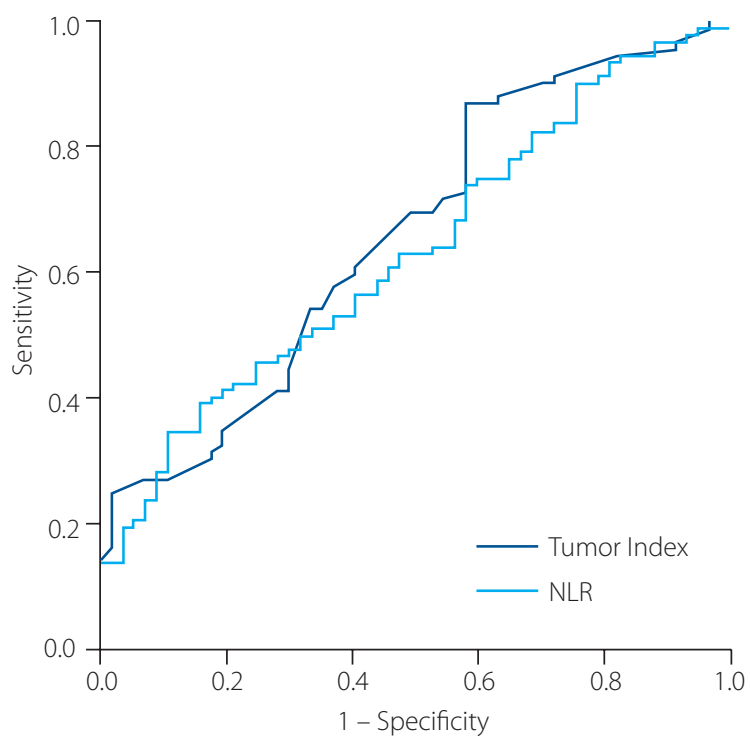

Figure 1. ROC curve plot for TI and NLR

Table II. Clinicopathological characteristics and lymph node metastases: univariate and multivariate analysis

\begin{tabular}{lcccc} 
& Univariate analysis & \multicolumn{2}{c}{ Multivariate analysis } \\
\hline Variable & $p$ value & OR & $95 \% \mathrm{Cl}$ & $p$ value \\
\hline Mixed-type by Lauren & 0.008 & 3.85 & $1.12-13.2$ & 0.033 \\
\hline Tumor stage & 0.001 & - & - & - \\
\hline Grading & 0.23 & - & - & - \\
\hline Patient age & 0.97 & - & - & - \\
\hline Tumor Index & $<0.001$ & 1.41 & $1.2-1.7$ & 0.004 \\
\hline NLR & 0.004 & - & $1.08-1.7$ & 0.009 \\
\hline PLR & 0.203 & - & - \\
\hline SIRI & 0.02 & - & - & - \\
\hline MLR & 0.388 & - & -
\end{tabular}


most effective in controlling nodal metastasis and results in increased $\mathrm{RO}$ resection rates, therefore it is recommended for patients with present lymph node involvement in preoperative assessment [7]. Although some experts recommend routine preoperative chemotherapy in all patients with stage $\geq$ Stage IB, many consider this as patient overtreatment, resulting in unnecessary risk and questionable clinical benefits. Initial diagnostic methods recommended for routine preoperative staging comprise of: blood tests, classic endoscopy with biopsy and computed tomography (CT), provide little information about lymph node status. Low sensitivity of CT 62.5 (91\% in systematic reviews) together with lack of global consensus on specific diagnostic criteria makes it a non-reliable source for nodal involvement evaluation [4, 10]. Multidetector-row computed tomography (MDCT), conventional magnetic resonance imaging (MRI), positron emission tomography (PET-CT) are additional imaging options available for clinicians, their value, however, varies between different studies and cannot distinctly confirm or exclude presence of nodal metastases in gastric cancer patients [10]. Endoscopic ultrasonography (EUS) provides powerful ability to assess depth of primary tumor invasion, but it is less useful in predicting lymph node status. Considering still relatively low availability of EUS in routine practice in many places of the word, its practical value in gastric cancer staging is lower than expected $[4,11,12]$.

In recent years several markers, derived from peripheral blood count levels, such as previously described NLR, PLR, MLR and SIRI, have been investigated and their prognostic value in many different cancers was reported. Few studies point out the connection between elevated PLR or NLR and presence of lymph node metastases in thyroid and gastric cancer $[24,25]$. To the best of our knowledge, our study is the first to investigate this relation in the European population of gastric cancer patients. Out of systemic inflammation markers considered in our analysis, only NLR was an independent factor associated with the lymph node invasion. As described, this may originate from the tumor's ability to create local metastasis by modulating local leukocyte differentiation, which leads to elevated neutrophil counts in peripheral blood [14-16, 19, 27]. There is no such documented association in platelets, which may explain why PLR, a widely reported prognostic factor, does not predict nodal metastasis. SIRI is a more complex marker incorporating monocyte, neutrophil and lymphocyte levels. While it is statistically significant as a single factor, we decided not to include it in multivariate analysis due to the correlation and lack of independency with NLR. NLR as a predictor explained more variation in lymph node status. In the recent years, some researchers investigated association between NLR or PLR and lymph node status. Zhang et al. concluded that both these markers are useful in predicting lymph node metastasis in gastric cancer patients [29]. Results of a similar study conducted by Pang et al. suggested the same conclusion [25]. Our study supports only one of these findings.
There are some limitations to this study. First of all, a retrospective design carried within a group of patients treated in a single hospital significantly restricts research possibilities and selection biases may exist. Secondly, several variables were obtained from postoperative pathology reports, which do not directly translate to preoperative setting. Finally, there might be underlying causes influencing values of peripheral blood counts, that were not recognized in routine clinical evaluation, and are outside of retrospective study design capabilities.

\section{Conclusions}

NLR and TI are available, reproducible, inexpensive markers and might be helpful with preoperative lymph node staging in patients with gastric cancer qualified for perioperative treatment. Combination of imaging techniques and simple markers derived from routine blood tests might preserve patients from unnecessary complications associated with neoadjuvant chemotherapy. There is a need for further prospective, large-scale research in this field.

\section{Conflict of interest: none declared}

This study was supported by grant no. NN403251940.

\section{Mateusz Pryt}

Medical University of Łódź

Department of Surgical Oncology

al. Kościuszki 4

90-419 Łódź, Poland

e-mail:mateuszpryt@gmail.com

\section{Received: 4 Dec 2018}

Approved for printing:20 Mar 2019

\section{References}

1. Torre LA, Bray F, Siegel RL et al. Global cancer statistics, 2012. CA Cancer JClin 2012; 65: 87-108.

2. Schwarz RE. Current status of management of malignant disease: current management of gastric cancer. J Gastrointest Surg 2015; 19: 782-8.

3. Kulig J, Wallner G, Drews $M$ et al. Polish consensus on treatment of gastric cancer; update 2017. Pol Przegl Chir 2017; 89: 59-73.

4. Smyth EC, Verheij M, Allum W et al. Gastric cancer: ESMO clinical practice guidelines for diagnosis, treatment and follow-up. Ann Oncol 2016; 27: 38-49.

5. Schuhmacher C, Gretschel S, Lordick F et al. Neoadjuvant chemotherapy compared with surgery alone for locally advanced cancer of the stomach and cardia: European organisation for research and treatment of cancer randomized trial 40954. J Clin Oncol 2010; 28: 5210-8.

6. Schirren R, Reim D, Novotny AR. Adjuvant and/or neoadjuvant therapy for gastric cancer? A perspective review. Ther Adv Med Oncol 2015; 7: 39-48.

7. Dong S, Yu J-R, Zhang Q et al. Neoadjuvant chemotherapy in controlling lymph node metastasis for locally advanced gastric cancer in a Chinese population. J Chemother 2016; 28: 59-64.

8. Li W, Qin J, Sun YH et al. Neoadjuvant chemotherapy for advanced gastric cancer: A meta-analysis. World J Gastroenterol 2010; 16: 5621-8.

9. Ronellenfitsch U, Schwarzbach M, Hofheinz R et al. Perioperative chemo(radio)therapy versus primary surgery for resectable adenocarcinoma of the stomach, gastroesophageal junction, and lower esophagus. Cochrane database Syst Rev 2013; (5): CD008107.

10. Kwee RM, Kwee TC. Imaging in assessing lymph node status in gastric cancer. Gastric Cancer 2009; 12: 6-22. 
11. Mocellin S, Marchet A, Nitti D. EUS for the staging of gastric cancer: A meta-analysis. Gastrointest Endosc 2011; 73: 1122-34.

12. Sharma M, Rai P, Rameshbabu CS. Techniques of imaging of nodal stations of gastric cancer by endoscopic ultrasound. Endosc Ultrasound 2014; 3: 179-90.

13. Grivennikov SI, Greten FR, Karin M. NIH Public Access. Cancer 2011; 140: 883-99.

14. Wculek SK, Malanchi I. Neutrophils support lung colonization of metastasis-initiating breast cancer cells. Nature 2015; 9: 528-413.

15. Mantovani A, Allavena P, Sica A et al. Cancer-related inflammation. Nature 2008; 23: 454-436.

16. Grivennikov $\mathrm{SI}$, Greten FR, Karin M. Immunity, inflammation, and cancer. Cell 2010; 140: 883-99.

17. Condeelis J, Pollard JW. Macrophages: Obligate partners for tumor cell migration, invasion, and metastasis. Cell 2006; 124: 263-6.

18. Huang $X$, Pan $Y$, Ma J et al. Prognostic significance of the infiltration of CD163+macrophages combined with CD66b + neutrophils in gastric cancer. Cancer Med 2018; 7: 1731-41.

19. Xu X, Yang C, Chen J et al. Interleukin-23 promotes the migration and invasion of gastric cancer cells by inducing epithelial-to-mesenchymal transition via the STAT3 pathway. Biochem Biophys Res Commun 2018; 499: 273-8.

20. Zheng $\mathrm{P}$, Luo $\mathrm{Q}$, Wang W et al.Tumor-associated macrophages-derived exosomes promote the migration of gastric cancer cells by transfer of functional Apolipoprotein E. Cell Death Dis 2018; 9.

21. Xiang J, Zhou L, Li X et al. Preoperative monocyte-to-lymphocyte ratio in peripheral blood predicts stages, metastasis, and histological grades in patients with ovarian cancer. Transl Oncol 2017; 10: 33-9.
22. Sun J, Chen X, Gao P et al. Can the neutrophil to lymphocyte ratio be used to determine gastric cancer treatment outcomes? A systematic review and meta-analysis. Dis Markers 2016; 2016: 7862469. doi: 10.1155/2016/7862469.

23. Geng Y, Zhu D, Wu C et al. A novel systemic inflammation response index (SIRI) for predicting postoperative survival of patients with esophageal squamous cell carcinoma. Int Immunopharmacol 2018; 65: 503-10.

24. Jiang K, Lei J, Chen $\mathrm{W}$ et al. Association of the preoperative neutrophil-tolymphocyte and platelet-to-lymphocyte ratios with lymph node metastasis and recurrence in patients with medullary thyroid carcinoma. Med (United States) 2016; 95: 1-6.

25. Pang W, Lou N, Jin C et al. Combination of preoperative platelet/lymphocyte and neutrophil/lymphocyte rates and tumor-related factors to predict lymph node metastasis in patients with gastric cancer. Eur J Gastroenterol Hepatol 2016; 28: 493-502.

26. Ohashi T, Komatsu S, Ichikawa D et al. Tumor Index as a combined indicator of tumor depth and size in gastric cancer. Anticancer Res 2016; 36: 1895-900.

27. Fluss R, Faraggi D, Reiser B. Estimation of the Youden Index and its associated cutoff point. Biometrical J 2005; 47: 458-72.

28. Ychou M, Boige V, Pignon J-P et al. Perioperative chemotherapy compared with surgery alone for resectable gastroesophageal adenocarcinoma: an FNCLCC and FFCD multicenter phase III trial. J Clin Oncol 2011; 29: 1715-21.

29. Fitzgibbons RJ, Giobbie-hurder A, Gibbs JO et al. Inguinal hernia in minimally symptomatic men. Jama 2006; 295: 285-93. 